\title{
Insulin analogues and cancer risk: the emergence of second-generation studies
}

\author{
A. G. Renehan
}

Received: 16 September 2011 / Accepted: 23 September 2011 /Published online: 28 October 2011

(C) Springer-Verlag 2011

\begin{abstract}
A number of observational studies have linked insulin glargine (A21Gly,B31Arg,B32Arg human insulin) with a putative increased cancer risk, particularly breast cancer, but many of these 'first generation' studies had study design and analysis flaws, and were inconclusive. A small number of 'second generation' studies are now emerging in which the applied pharmaco-epidemiological principles are more robust. For example, when Ruitar and colleagues (Diabetologia DOI:10.1007/s00125-0112312-4) focused specifically on breast cancer rather than all incident cancer risk, they were able to show a positive association with insulin glargine for breast cancer although there was no association with all incident cancer risk. A list of preferred qualities for pharmaco-epidemiological studies is presented.
\end{abstract}

Keywords Diabetes · Cancer risk · Glucose-lowering drugs $\cdot$ Insulin analogue $\cdot$ Pharmaco-epidemiology

\section{Abbreviation \\ GPRD General Practice Research Database}

\footnotetext{
A. G. Renehan $(\varangle)$

School of Cancer and Enabling Sciences,

University of Manchester,

Manchester Academic Health Science Centre,

The Christie NHS Foundation Trust,

Wilmslow Road,

Manchester M20 4BX, UK

e-mail: arenehan@picr.man.ac.uk
}

Readers of Diabetologia will be familiar with the four papers [1-4] published simultaneously in the journal in September 2009 that linked the long-acting insulin analogue glargine (A21Gly,B31Arg,B32Arg human insulin) with a putative increased risk of cancer incidence, notably breast cancer. These papers awakened an unprecedented debate among the diabetes community of the benefit-risk profile of insulin analogues [5]. The four papers and preceding publications formed a group of 'first generation' studies that were justifiably criticised [6] and were without argument, inconclusive. However, these papers served as a trigger to initiate research activities across cancer and diabetes disciplines (where previously there had been very little cross-fertilisation), to raise political awareness across many stakeholders, and to focus attention on the methodological issues underpinning the pharmaco-epidemiological studies in this field.

Immediately after the summer of 2009 , not unexpectedly, there was a rush by many research teams to explore their databases to refute or confirm the findings of the four Diabetologia papers. Unfortunately, several of these post2009 publications suffered with flaws - some openly exposed [7] - and they served only to perpetuate the inconclusiveness. Fortunately, a small number of studies have recently emerged that take us to a new 'second generation' of studies. It is important to re-iterate that it is generally accepted that in pursuing the hypothesised link between insulin analogues and cancer risk, randomised trials are neither practical nor feasible, and thus we have to rely on observational data [8]. To this end, a list of preferred design features of a second generation study is 
shown in the text box. Importantly, cancer is not a single disease and it is imperative that site- and sex-specific cancers are stated as primary (not secondary) endpoints in protocols.

\section{Insulin analogues and cancer risk: preferred design features for a pharmaco-epidemiological study}

- Site- and sex-specific cancer endpoints

- Cohort design: case-control design may be used but can only analyse one cancer type at a time

- Cancer incidence: preferred over cancer mortality; the latter endpoint has many potential confounders such as stage at presentation and treatment selection

- Cancer ascertainment: standardised and validated source

- Incident drug exposure

- Time-varying analysis of exposure

- Inclusion of key covariates, e.g. smoking and body mass index

- Latent period: biologically plausible time period between exposure and cancer event

- Detection bias: check for reverse causation

- Avoidance of immortal time bias: include information from before exposure

- Dose: preference for defined daily dose with ability to convert to an equivalent dose if required

- Methods to minimise confounding:

-Adherent patient analyses

-Matching e.g. propensity score analysis

-Instrumental variable analyses

- Multiple sensitivity analyses - for example:

-Fixed versus as-treated analysis

-Varying the 'tail-off' on the exposure

In the present issue of Diabetologia, Ruitar and colleagues [9] tackle the hypothesis that incident cancer risk is increased among patients with type 2 diabetes on either insulin glargine, other insulin analogues or human insulin. Using the PHARMO Record Linkage System from the Netherlands, they report a very comprehensive set of analyses, which ticks many of the preferred qualities shown in the text box. As an example, the authors dealt with the insulin exposure using time-varying approaches; performed fixed and as-treated analyses; undertook multiple sensitivity analyses around dosage; addressed the potential confounding of reverse causality and detection bias [10]; reported patient adherence data; and attempted to minimise residual confounding through a propensity score analysis. After all these considerations, the headline finding remained the same - that insulin glargine and other insulin analogues have a lower risk of cancer than human insulin. At first glance, this seems at odds with the authors' original hypothesis and may be misleading to the cursory reader. However, all cancer risk is a composite endpoint, and interpreting associations with it is near meaningless.

Taking a more focused endpoint in their secondary outcome analyses, the authors reported a significant positive association between insulin glargine and breast cancer risk: in the as-treated analysis there was a doseresponse effect. This echoes the findings from the original Scottish [1] and Swedish [2] data. It is also consistent with the recently published analysis by Suissa and colleagues [11], who used the UK's General Practice Research Database (GPRD) to identify a cohort of women with type 2 diabetes treated with insulins and followed until first breast cancer diagnosis. They used a matching process of users of insulin glargine with users of other insulins on age, calendar time and duration of prior insulin use, and calculated HRs, and thus also ticked many of the preferred qualities listed in the text box. In their time-varying analysis, they showed that insulin glargine use was not associated with an increased risk of breast cancer during the first 5 years of use, but instead risk tended to increase after 5 years (HR $1.8 ; 95 \%$ CI $0.8,4.0$ ), and significantly so for the women who had been on insulin before starting insulin glargine (HR 2.7; 95\% CI 1.1, 6.5).

Future studies are needed to replicate these findings, stratify results into pre- and post-menopausal breast cancer, and ultimately, stratify by histological sub-classifications (for example oestrogen and progesterone receptor status). This will require linkage with validated and standardised cancer data - for instance, tumour registry linkages were not used in the Dutch [9] and GPRD [11] studies. The inclusion of key covariates - such as body mass index and smoking, and in the example of breast cancer, screening and hormonal replacement therapy use - will be required, as these are effect-modifiers of associations between obesity and cancer [12] and may equally apply to diabetes-cancer associations.

As the methodology improves and second generation studies emerge, there is a clear requirement for an additional dimension, namely a multi-disciplinary research team. Expertise in pharmaco-epidemiology is a core pre-requisiteadditional contributory members should include: bioinformatics to support the increasing computational demands of analyses; tumour registry expertise; clinical expertise in diabetes and oncology; and tumour scientists to unscramble the biological plausibility of the observed findings. Finally, the multi-disciplinary approach is required for the conversion 
of these new data into clinically meaningful guidance. As an example, after decades of debate, oncologists now can accurately inform women regarding cancer risk before commencement of hormone replacement therapy. These quality standards are the clinical targets for the near future so that millions of patients with diabetes can benefit from their glucose-lowering treatments with minimal risk of adverse neoplastic consequences.

Acknowledgements I acknowledge the many discussions with learned international colleagues on the methodologies underpinning analyses on this topic.

Contribution statement AGR was responsible for the interpretation of data and literature, wrote and revised the article, and gave approval of the final version to be published.

Duality of interest A.G. Renehan has served on the advisory board of and received research support from Novo Nordisk. He is the European Cancer Organisation representative on the EASD Diabetes and Cancer Research Task Force and a member of the Diabetes and Cancer Research Consortium (DCRC). The views expressed in this commentary are not necessarily those held by the EASD or the DCRC.

\section{References}

1. Colhoun HM, SDRN Epidemiology Group (2009) Use of insulin glargine and cancer incidence in Scotland: a study from the Scottish Diabetes Research Network Epidemiology Group. Diabetologia 52:1755-1765, Erratum 52: 2469
2. Jonasson JM, Ljung R, Talback M, Haglund B, Gudbjornsdottir S, Steineck G (2009) Insulin glargine use and short-term incidence of malignancies - a population-based follow-up study in Sweden. Diabetologia 52:1745-1754

3. Currie CJ, Poole CD, Gale EA (2009) The influence of glucoselowering therapies on cancer risk in type 2 diabetes. Diabetologia 52:1766-1777

4. Hemkens LG, Grouven U, Bender R et al (2009) Risk of malignancies in patients with diabetes treated with human insulin or insulin analogues: a cohort study. Diabetologia 52:1732-1744

5. Smith U, Gale EA (2009) Does diabetes therapy influence the risk of cancer? Diabetologia 52:1699-1708

6. Pocock SJ, Smeeth L (2009) Insulin glargine and malignancy: an unwarranted alarm. Lancet 374:511-513

7. Carstensen B (2010) Comment on: Yang et al. (2010) Associations of hyperglycemia and insulin usage with the risk of cancer in type 2 diabetes: the Hong Kong Diabetes Registry. Diabetes 59:1254-1260. doi:10.2337/db10-0777, Diabetes 59: e17-18

8. Renehan A, Smith U, Kirkman MS (2010) Linking diabetes and cancer: a consensus on its complexity [commentary]. Lancet $375: 2201-2202$

9. Ruiter R, Visser LE, van Herk-Sukel MPP et al (2011) Risk of cancer in patients on insulin glargine and other insulin analogues in comparison with those on human insulin: results from a large population-based follow-up study. Diabetologia. doi:10.1007/ s00125-011-2312-4

10. Johnson JA, Bowker SL, Richardson K, Marra CA (2011) Timevarying incidence of cancer after the onset of type 2 diabetes: evidence of potential detection bias. Diabetologia 54:2263-2271

11. Suissa S, Azoulay L, Dell'aniello S, Evans M, Vora J, Pollak M (2011) Long-term effects of insulin glargine on the risk of breast cancer. Diabetologia 54:2254-2262

12. Renehan AG, Soerjomataram I, Leitzmann MF (2010) Interpreting the epidemiological evidence linking obesity and cancer: a framework for population-attributable risk estimations in Europe. Eur J Cancer 46:2581-2592 